\title{
Avaliação de impacto da ONG Refazer no tratamento médico de crianças em risco social
}

\author{
Ana Maria Magalhães Costa, ${ }^{1}$ Kátia da Silva, ${ }^{1}$ Saint Clair Gomes Jr., ${ }^{1}$ \\ Maria Inês de Oliveira, ${ }^{2}$ Rosane de Mello, ${ }^{1}$ Márcia de Carvalho ${ }^{3}$ \\ e Maria Auxiliadora Gomes ${ }^{1}$
}

Com citar

Costa AMM, Silva K, Gomes Jr SC, Oliveira MI, Mello R, Carvalho M, et al. Avaliação de impacto da ONG Refazer no tratamento médico de crianças em risco social. Rev Panam Salud Publica. 2011:30(3): 231-9.

RESUMO Objetivo. Avaliar o impacto das ações de apoio ao tratamento médico de crianças em risco social realizadas pela Organização Não Governamental Refazer.

Métodos. Foi realizado um estudo longitudinal com 77 pacientes do Rio de Janeiro em tratamento no Instituto Fernandes Figueira e apoiados pela Refazer. O período de avaliação foi de dois anos, sendo um anterior e outro na vigência da intervenção (apoio da ONG). Foram comparados o percentual de internação, o tempo médio, a gravidade, a utilização de tecnologia, número e condições clínicas de consultas ambulatoriais.

Resultados. Os indicadores relacionados às internações identificaram mudanças ocorridas durante a intervenção. Houve uma redução do tempo médio e do percentual de internação. As patologias mais frequentes foram as malformações congênitas e anomalias cromossômicas e as doenças do sistema nervoso. Estas doenças e a HIV/AIDS mostraram-se mais prevalentes no grupo de crianças que continuou apresentando o desfecho internação mesmo após receber o apoio da ONG. Conclusões. A análise dos indicadores mostrou que os resultados estão adequados à missão da ONG e a população assistida apresenta um perfil que se beneficia com a inserção na rede de apoio social. Apesar da impossibilidade em se afirmar que os benefícios encontrados são exclusivos das ações da ONG, desenvolveu-se uma avaliação de adequação simples e de fácil aplicação pelas próprias ONGs, o que representa um avanço na direção da institucionalização de avaliações pelas ONGs que atuam na saúde.

Palavras chaves Organizações não governamentais; avaliação de programas e projetos de saúde; criança; hospitalização; Brasil.

A segunda metade do século XX assistiu à expansão de organizações não go-

\footnotetext{
Fundação Oswaldo Cruz (FIOCRUZ), Instituto Fernandes Figueira, Unidade de Pesquisa Clínica, Rio de Janeiro (RJ), Brasil. Enviar correspondência a: Ana Maria Magalhães Costa, magcosta@iff. fiocruz.br

2 Universidade Federal Fluminense (UFF), Instituto da Saúde da Comunidade, Departamento de Epidemiologia e Bioestatística, Niterói (RJ), Brasil.

3 FIOCRUZ, Escola Nacional de Saúde Pública Epidemiologia e Métodos Quantitativos, Rio de Janeiro (RJ), Brasil.
}

vernamentais (ONGs) promovida por mudanças ocorridas no período. De um lado, a reforma do Estado, que fortaleceu seu papel na formulação de políticas e diminuiu sua presença direta nas ações sociais; do outro, a mobilização da sociedade civil, disposta a participar efetivamente das questões de interesse público e de caráter social. Foi neste contexto que essas organizações ganharam reconhecimento, atuando como parceiros dos seto- res público e privado em diferentes áreas, entre elas a da saúde, particularmente a da criança.

No desenvolvimento de ações para melhorar a saúde infantil, as ONGs têm participado da elaboração da agenda de prioridades de programas de saúde, e têm realizado atividades de pesquisa, educação e assistência, promovendo a capacitação de profissionais e agentes de saúde e se envolvendo com a gestão de 
projetos e serviços de saúde (1). Grande parte destas atividades é similar às ações realizadas na área de saúde pública.

Nos anos 90, observou-se no Brasil a retomada do papel do Estado na garantia dos direitos do cidadão. Este período foi marcado pelo avanço das políticas sociais (aumento real do salário mínimo, transferência de renda, segurança alimentar e disponibilidade de crédito popular e agrícola) e da efetividade da política de saúde na redução da mortalidade infantil, pelo controle das deficiências nutricionais e das doenças infecciosas. No entanto, estas políticas não têm sido suficientes para atender as demandas sociais e de saúde de grupos mais vulneráveis. É neste cenário que se destaca a atuação das ONGs.

Apesar do crescimento das atividades das ONGs, sabe-se pouco sobre o impacto de suas intervenções e programas na população beneficiada. A dificuldade em se estabelecer um desenho de estudo com rigor metodológico, necessário para estas avaliações, explica o pequeno número de estudos nessa área. Uma revisão sistemática realizada nos relatórios produzidos para o Fundo das Nações Unidas para a Criança (UNICEF) mostrou que 15\% destes estudos incluíam avaliação de impacto, mas devido à fragilidade dos métodos aplicados poucos apresentavam resultados consistentes (2). Outra revisão sobre as ações das ONGs na saúde da criança encontrou 21\% (12/57) de artigos que mencionavam aspectos relacionados ao impacto na população (1). O conhecimento sobre o impacto resultante de um programa é fundamental para a tomada de decisões quanto à continuidade e sustentabilidade dos projetos, assim como para a aplicação dos recursos destinados aos mesmos.

As intervenções de promoção de saúde e prevenção de doenças, principais programas desenvolvidos pelas ONGs que atuam na saúde da criança, ocorrem, na sua maioria, num contexto de desigualdade social. Fatores diversos, desde os relacionados à distribuição de renda e as políticas de saúde, até as condições de nascimento de cada indivíduo, podem afetar os resultados das ações. Isso torna as cadeias causais muito longas e complexas e dificulta a adoção de modelos de eficácia nas avaliações destes programas (3). Devido a estas características, a investigação da efetividade tem sido indicada como mais adequada para avaliar este tipo de intervenção.
No Brasil, uma rede composta por 23 ONGs chamada Rede Saúde Criança (4) está associada a hospitais públicos e tem como objetivo melhorar as condições de saúde de crianças em risco social e que são atendidas nestes hospitais. Como parceiras de órgão público, espera-se que estas ONGs alinhem-se ao esforço do Ministério da Saúde (5) no sentido de institucionalizar a prática de avaliação de seus programas e iniciativas (6), incorporando tal prática à rotina de seus processos.

O objetivo deste trabalho foi identificar indicadores sensíveis às mudanças promovidas pela intervenção de uma ONG que apóia crianças em situação de risco em tratamento numa unidade pública de saúde materno infantil. Esperase que os indicadores identificados possam ser utilizados nas avaliações das ONGs contribuindo como ferramenta de tomada de decisão e re-direcionamento das ações quando necessário.

\section{MÉTODOS}

O Instituto Fernandes Figueira (IFF) é uma unidade de assistência terciária da Fundação Oswaldo Cruz e um centro de referência nacional para o Ministério da Saúde na área de atenção à mulher, criança e adolescente (7). O Instituto está inserido no Sistema Único de Saúde (SUS). O SUS representa o sistema público brasileiro e tem como princípio o acesso universal e integral da população aos serviços de saúde. O sistema atende uma população de baixa renda proveniente de diversos municípios do país, incluindo aqueles do Estado do Rio de Janeiro.

A ONG Refazer apóia crianças e adolescentes em tratamento no hospital do IFF. É uma instituição da sociedade civil, de utilidade pública, sem fins lucrativos e sem filiação partidária ou religiosa. Sua missão é "evitar a re-internação e/ou agravamento das doenças das crianças e adolescentes encaminhados pelo IFF, disponibilizando meios que promovam a melhoria da qualidade de vida de suas famílias" (8).

Os critérios para a inclusão das crianças nos projetos sociais da ONG se baseiam principalmente na renda familiar, que deve ser de até hum salário mínimo (equivalente a US\$ 344,93). Além disso, elas devem possuir condição de vulnerabilidade para a adesão ao tratamento, tais como baixo nível socio- econômico, dificuldade de acesso aos bens e serviços e às políticas públicas, condições precárias de moradia e baixa escolaridade dos responsáveis, também são consideradas. As crianças são encaminhadas a Refazer pelo serviço social do IFF após avaliação e constatação da situação de risco vivida pelas famílias.

Foi realizado um estudo longitudinal, retrospectivo, que analisou os indicadores de utilização de serviços hospitalares relacionados a consultas ambulatoriais, internações e uso de tecnologia em crianças sob tratamento no IFF e atendidas pela Refazer. A intervenção analisada foi o apoio desenvolvido pela ONG. Compararam-se os dados obtidos em prontuários hospitalares referentes a dois períodos, o ano precedente ao vínculo com a ONG e o ano durante o qual a criança recebeu algum benefício da ONG.

A população do estudo foi constituída por crianças e adolescentes em condição de vulnerabilidade, em tratamento no IFF. Como critérios de inclusão no estudo consideraram-se:

1) ingresso na ONG entre 01 de janeiro de 2003 e 08 de novembro de 2007.

2) mínimo de um ano de tratamento na unidade hospitalar antes de se vincular à ONG.

Como a hipótese foi de que crianças em situação de vulnerabilidade têm a evolução de sua doença amenizada e a necessidade de utilização de serviços hospitalares reduzida quando recebem apoio complementar ao tratamento, foram excluídos os pacientes que participavam de projetos de pesquisa do hospital cujos protocolos de monitoramento dos sujeitos previssem uma utilização diferenciada de serviços hospitalares em relação às rotinas, o que poderia provocar um viés na avaliação.

A fonte de informação para identificação dos pacientes foi o registro dos mesmos na ONG. Todos os indivíduos que preencheram os critérios de inclusão foram selecionados para o estudo. Os dados foram coletados no período de janeiro a outubro de 2009 de acordo com os prontuários dos pacientes e complementados com o levantamento das fichas da ONG.

$\mathrm{O}$ acesso aos documentos foi realizado com as autorizações do chefe do Departamento de Informação, responsável pela guarda dos prontuários no hospital, 
e da presidente da Refazer. Como não houve entrevistas realizadas diretamente com os pacientes ou seus responsáveis não foi exigido pelo Comitê de Ética (CEP) o Termo de Consentimento Livre e Esclarecido (TCLE). O estudo foi aprovado pelo CEP do IFF/FIOCRUZ em 27/07/2007, registrado com o número de cadastro FR 129040 e CAAE 0014.0.008.000-07.

As atividades da ONG se constituíram em apoio diretamente relacionado à assistência à saúde que se traduziu em empréstimos de aparelhos respiratórios (BIPAP e concentrador de $\mathrm{O}_{2}$ ) doação de medicamentos, cadeira de rodas e insumos hospitalares (espaçador, aerocâmara, nebulizador, aspirador, balão de $\mathrm{O}_{2}$, sondas, luvas, seringas, gazes e bolsas de colostomia). Este apoio se baseou na prescrição terapêutica feita pelos profissionais de saúde do IFF. O fornecimento de leite, cesta básica e auxíliotransporte para comparecimento às atividades realizadas na sede da ONG foi considerado apoio indiretamente relacionado à assistência à saúde. Para este tipo de apoio, os critérios foram estabelecidos pela ONG.

Para avaliar a capacidade da ONG em responder às demandas dos profissionais para o tratamento das crianças e adolescentes selecionados analisou-se o percentual de pacientes com recomendações atendidas em relação ao total de pacientes com recomendações prescritas.

$\mathrm{O}$ perfil demográfico e sócio-econômico da população foi traçado a partir das seguintes características: sexo; faixa etária (0-4 ; 5 a 9; 10 a 14 e 15-16 anos); cor branca e negra, esta última incluindo pretos e pardos (9); condições de moradia (se tem ou não água encanada e/ou sistema de esgoto); grau de escolaridade do responsável (<ensino fundamental, ensino fundamental completo, ensino médio, universitário completo) e renda familiar (<1, entre 1 e 1,9; 2 e 2,$9 ; 3$ e 3,9 e 4 ou mais salários mínimos).

Também foram observados os benefícios recebidos pela criança através de programas de renda mínima, tais como bolsa família, bolsa escola, cheque cidadão e o benefício de prestação continuada (BPC) (10).

O diagnóstico principal, descrito no prontuário, foi agrupado de acordo com a Classificação Internacional de Doenças e Problemas Relacionados à Saúde (CID) -10a revisão (11), considerando os três primeiros caracteres do código. No caso de associação de patologias, optou-se por classificar pelo diagnóstico principal. As datas de abertura de prontuário no IFF e de entrada no Refazer foram utilizadas para o cálculo do tempo de tratamento.

Os desfechos do estudo foram os indicadores de utilização de serviços hospitalares. Foi feita a comparação dos seguintes indicadores:

1) ambulatoriais - número de consultas, percentual de consultas sem sintomas, percentual de consultas com sintomas relacionados à doença de base, percentual de consultas com relato de diarréia e/ou pneumonia - considerados "eventos sentinela" (12)

2) de internação - total de internações, total de dias internados e média dos dias internados, percentual de internados.

3) de gravidade do quadro clínico percentual de internações na unidade de pacientes graves (UPG) e/ou de utilização de tecnologias, tais como: acesso venoso central, associação de 2 ou mais antimicrobianos, assistência ventilatória, expansor, hemoderivados, nutrição parenteral, procedimento cirúrgico, suplemento de oxigênio $(\mathrm{O} 2)$ e traqueostomia.

Para a avaliação dos desfechos foram excluídos os óbitos ocorridos durante o período do estudo, uma vez que sua ocorrência poderia contribuir para subestimar os indicadores no período pós intervenção. Para conhecer as características em relação à internação hospitalar nos dois períodos (antes e depois da intervenção da $\mathrm{ONG}$ ), a população de estudo foi categorizada em 4 grupos: 1-crianças que não internaram ao longo do período do estudo, 2-crianças que internaram somente no período que precedeu a intervenção, 3-crianças que internaram apenas após a intervenção, 4crianças que internaram em ambos os períodos. Os grupos 3 e 4 constituíram um único grupo na análise multivariada (grupo 3+4). Um modelo logístico investigou a associação da internação hospitalar com o tempo de tratamento, a idade e a CID10 (11). Em relação aos diagnósticos, foram definidos quatro grupos de morbidade segundo a CID10 (11) : B HIV/AIDS, G — doenças do sistema nervoso, $\mathrm{Q}$ - malformações congênitas, deformidades e anomalias cromossômicas e outros que reuniu as demais pato- logias identificadas na amostra. Apesar das doenças respiratórias apresentarem uma alta prevalência na população, no grupo $3+4$ a sua frequência foi igual a zero, e por isso foram excluídas do modelo. Utilizou-se o modelo de regressão logística multinominal.

O grupo 1 foi utilizado como referência para a análise uma vez que não apresentou nenhuma internação durante o período de observação, sendo considerado o grupo de menor risco para os desfechos selecionados. A avaliação de adequação das ações da ONG foi realizada a partir da análise dos indicadores nos dois períodos.

O banco de dados utilizado foi o EpiInfo versão 3.5.1 de 2008 e o SPSS versão 15.0. Aplicaram-se os testes não paramétricos de Wilcoxon e Mc Nemar para análise de amostras pareadas, para comparação dos valores médios e proporções dos indicadores respectivamente.

\section{RESULTADOS}

A análise dos registros da Refazer identificou 82 pacientes elegíveis. Após a aplicação dos critérios de exclusão, cinco foram retirados do estudo devido à participação em projetos de pesquisa do IFF, restando 77 pacientes.

A população do estudo foi constituída por crianças e adolescentes, a maioria do sexo masculino $(58 \%)$, com idade abaixo de 10 anos $(76,7 \%)$ e de cor negra $(62,3 \%)$. Apresentou um baixo nível sócio econômico refletido no grau de instrução da mãe ou responsável, na renda familiar e nas condições de moradia. $\mathrm{O}$ ensino fundamental foi a escolaridade informada de $79 \%$ das mães ou responsáveis, porém a maioria não completou esta etapa do ensino. Mais da metade das famílias tinha renda abaixo de dois salários mínimos $(58,1 \%)$ e $20,8 \%$ dos pacientes estavam assegurados pelo BPC, direito garantido pela Constituição Federal de 1988 gerido pelo Ministério do Desenvolvimento Social e Combate à Fome (10). O BPC consiste no pagamento de um salário mínimo mensal a pessoas com deficiência incapacitante para a vida independente e para o trabalho e cuja renda per capita familiar seja inferior a $1 / 4$ do salário mínimo. Os dados sobre benefícios oriundos de programas de renda mínima não foram registrados e/ou informados, o que inviabilizou uma análise da população beneficiada por estes programas. A maior parte dos 
domicílios $(83,1 \%)$ era provida de água e esgoto, 5,6\% permaneciam carentes de saneamento básico (tabela 1).

As crianças e adolescentes eram provenientes da região metropolitana do Rio de Janeiro, principalmente do município do Rio de Janeiro $(66,2 \%)$ e da Baixada Fluminense $(31,2 \%)-14,3 \%$ de Duque de Caxias, 5,2\% de Belford Roxo, 3,9\% de Nova Iguaçu, 3,9\% de São João de Meriti e 3,9\% de outros. Os restantes $(2,6 \%)$ eram oriundos de municípios do entorno.

A capacidade da Refazer em atender às recomendações da equipe de saúde do IFF pode ser analisada na tabela 2. Nos itens diretamente relacionados à assistência, o percentual de respostas às recomendações ficou acima de $80 \%$, com exceção da demanda por cadeira de rodas $(70,0 \%)$. Nestes casos os pacientes foram encaminhados a programas oficiais do estado para obtenção do equipamento. Os 77 pacientes do estudo eram, em sua maioria, portadores de doenças crônicas entre as quais se destacaram as malformações congênitas, deformidades

TABELA 1. Características sócio-demográficas de crianças em risco social em tratamento no Instituto Fernandes Figueira, Rio de Janeiro, Brasil e apoiadas pela ONG Refazer durante 0 período de 2003 à 2007

\begin{tabular}{|c|c|c|}
\hline Característica & No. & $\%$ \\
\hline \multicolumn{3}{|l|}{$\operatorname{Sexo}(n=77)$} \\
\hline Feminino & 32 & 41,6 \\
\hline Masculino & 45 & 58,4 \\
\hline \multicolumn{3}{|l|}{ Idade (anos) $(n=77)$} \\
\hline $0-4$ & 27 & 35,1 \\
\hline $5-9$ & 32 & 41,6 \\
\hline $10-14$ & 12 & 15,0 \\
\hline $15-16$ & 6 & 78,0 \\
\hline \multicolumn{3}{|l|}{ Cor $(n=77)$} \\
\hline Negra (pretos e pardos) & 48 & 62,3 \\
\hline Branca & 29 & 37,7 \\
\hline \multicolumn{3}{|l|}{ Condições de Moradiaa ${ }^{a}$} \\
\hline Com água $(n=73)$ & 67 & 91,8 \\
\hline Com esgoto $(n=71)$ & 59 & 83,1 \\
\hline Sem água e esgoto $(n=71)$ & 4 & 56,0 \\
\hline \multicolumn{3}{|l|}{ Renda Familiar $(n=62)$} \\
\hline < 1 salário mínimo & 6 & 97,0 \\
\hline Entre 1 e 1,9 salários mínimos & 30 & 48,4 \\
\hline Entre 2 e 2,9 salários mínimos & 15 & 24,2 \\
\hline Entre 3 e 3,9 salários mínimos & 7 & 11,3 \\
\hline > 4 salários mínimos & 4 & 64,0 \\
\hline \multicolumn{3}{|l|}{$\begin{array}{l}\text { Escolaridade do Responsável } \\
\qquad(n=62)\end{array}$} \\
\hline$<$ Ensino Fundamental & 32 & 51,6 \\
\hline Ensino Fundamental completo & 17 & 27,4 \\
\hline Ensino Médio completo & 12 & 19,4 \\
\hline Ensino Universitário completo & 1 & 16,0 \\
\hline
\end{tabular}

e anomalias cromossômicas $(32,5 \%)$, as doenças do sistema nervoso $(18,2 \%)$ e as doenças do aparelho respiratório $(16,9 \%)$ (tabela 3).

No estudo, três crianças $(3,9 \%)$ evoluíram para óbito, duas eram portadoras de vírus da imunodeficiência humana (HIV/AIDS) (10 e 17 anos) e uma apresentava anomalia cromossômica (14 anos). Estes óbitos ocorreram até dez meses após a entrada na Refazer.

$\mathrm{Na}$ análise dos indicadores de utilização de serviços hospitalares, constatou-se uma redução dos indicadores relacionados às internações (Tabela 4), porém apenas para o percentual de crianças internadas $(P=0,02)$ e para o tempo médio de internação $(P=0,016)$ houve uma diferença estatisticamente significativa. A gravidade clínica, medida a partir do percentual de internações na UPG e do uso de tecnologias durante as internações, não foi estatisticamente significante. A análise de sensibilidade realizada com os 77 pacientes não alterou o significado estatístico dos resultados.

Houve mais referências às internações fora do IFF no período anterior $(23 \%)$ do que durante a intervenção (12,2\%), sendo esta diferença estatisticamente significativa $(P=0,03)$.

Os indicadores selecionados para a análise da utilização de serviços ambulatoriais refletiram se as consultas estavam relacionadas à doença de base, à rotina de acompanhamento ou a algum evento sentinela (tabela 4). A comparação destes indicadores nos dois perío-

TABELA 2. Tipo de apoio da Refazer à assistência a saúde relacionado com a recomendação prescrita e a capacidade de atendimento às crianças e aos adolescentes em risco social em tratamento no Instituto Fernandes Figueira (IFF), Rio de Janeiro, Brasil, durante o período de 2003 à 2007

\begin{tabular}{|c|c|c|c|}
\hline \multirow[b]{3}{*}{ Apoio da Refazer } & \multicolumn{3}{|c|}{ Recomendação da IFF } \\
\hline & \multirow{2}{*}{$\frac{\text { Prescrita }}{\text { No. }}$} & \multicolumn{2}{|c|}{ Atendida } \\
\hline & & No. & $\%^{\mathrm{a}}$ \\
\hline \multicolumn{4}{|l|}{ Direto } \\
\hline Medicamento & 45 & 40 & 89 \\
\hline Insumos hospitalares & 16 & 13 & 81 \\
\hline Aparelhos respiratórios & 2 & 2 & 100 \\
\hline Cadeira de rodas & 10 & 7 & 70 \\
\hline \multicolumn{4}{|l|}{ Indireto } \\
\hline Leite & 22 & 19 & 86 \\
\hline Cesta básica & 18 & 16 & 89 \\
\hline Vale transporte & 3 & 3 & 100 \\
\hline
\end{tabular}

dos não apresentou diferenças estatísticas significativas.

Em ambos os períodos, o ambulatório do serviço de Pediatria foi o que atendeu o maior percentual de crianças da população $(60,0 \%)$. No período anterior à intervenção, procuraram atendimento ambulatorial em outras unidades de saúde $23,0 \%$ dos pacientes e no período da intervenção $21,6 \%$, redução sem significância estatística $(p=0,82)$.

A análise de cada grupo em relação às internações hospitalares nos dois períodos mostrou que os pacientes do grupo $3+4$, cuja evolução foi menos favorável, apresentaram uma menor média de idade e de tempo de tratamento no IFF, este último influenciado, provavelmente, pela idade. Este grupo apresentou também uma diferença na prevalência das doenças do sistema nervoso, das malformações congênitas, deformidades e anomalias cromossômicas, e HIV/ AIDS quando comparado com o grupo que não teve internações (Tabela 5). Embora as doenças respiratórias fossem a terceira morbidade mais frequente na população do estudo, este diagnóstico não esteve presente entre as crianças que internaram após a intervenção.

$\mathrm{Na}$ análise multivariada, a variável referente ao perfil de morbidade - malformações congênitas e anomalias cromossômicas (OR = 5,1; IC95\% 1,2-22,6), doenças do sistema nervoso $(\mathrm{OR}=8,7$; IC95\% 1,5-49,8) - apresentou uma associação estatística significativa com o desfecho internação "depois" (grupo 3+4) quando comparado com o desfecho referente à "não internação" durante os dois períodos do estudo (grupo 1). Para a $\mathrm{HIV} / \mathrm{AIDS}$ (OR = 9,8; IC95\% 0,9-110,9; $P=0,07)$ o significado estatístico desta associação foi limítrofe. Em relação ao tempo de tratamento e a idade não houve diferenças estatisticamente significativas na comparação dos grupos 2 e $3+4$ com o grupo 1, de referência.

\section{DISCUSSÃO}

A incorporação pela ONG de uma prática sistemática de avaliação de resultados baseada em indicadores e inferências é importante para a legitimidade e reconhecimento de seu trabalho. O presente estudo traz uma metodologia de análise da adequação das ações de apoio da ONG Refazer com a sua missão e sugere indicadores para compor as avaliações de atividades realizadas por 
TABELA 3. Diagnósticos principais das crianças e dos adolescentes em risco social atendidas no Instituto Fernandes Figueira, Rio de Janeiro, Brasil, e assistidas pela Refazer, durante o período de 2003 a 2007 de acordo com a Classificação Internacional de Doenças e Problemas Relacionados à Saúde (CID)

\begin{tabular}{|c|c|c|c|}
\hline \multirow{2}{*}{$\begin{array}{l}\text { CID } \\
\text { capítulo }\end{array}$} & \multirow[b]{2}{*}{ Diagnóstico } & \multicolumn{2}{|c|}{ Criancas e adolescentes ${ }^{2}$} \\
\hline & & No. & $\%$ \\
\hline Q & $\begin{array}{l}\text { Malformações congênitas, deformidades e anomalias } \\
\text { cromossômicas } \\
\text { Malformações múltiplas (11) } \\
\text { Malformações do sistema nervoso (6) } \\
\text { Hidrocefalia (2) } \\
\text { Espinha bífida (2) } \\
\text { Microcefalia (1) } \\
\text { Síndrome de Arnold-Chiari (1) } \\
\text { Síndrome de Down (4) } \\
\text { Malformação do aparelho digestivo (2) } \\
\text { Megacolon congênito (1) } \\
\text { Anomalia ano-retal (1) } \\
\text { Ictiose congênita (2) }\end{array}$ & 25 & 32,5 \\
\hline G & $\begin{array}{l}\text { Doenças do sistema nervoso } \\
\text { Encefalopatias (9) } \\
\text { Neuropatias (2) } \\
\text { Outros quadros convulsivos (2) } \\
\text { Ataxia-teleangiectasia (1) }\end{array}$ & 14 & 18,2 \\
\hline J & $\begin{array}{l}\text { Doenças do aparelho respiratório } \\
\text { Doença pulmonar obstrutiva crônica (10) } \\
\text { Pneumonia e/ou sinusite (2) } \\
\text { Rinite alérgica (1) }\end{array}$ & 13 & 16,9 \\
\hline$A-B$ & $\begin{array}{l}\text { Doenças infecciosas e parasitárias } \\
\text { HIV (8) }\end{array}$ & 8 & 10,4 \\
\hline E & $\begin{array}{l}\text { Doenças endócrinas, nutricionais e metabólicas } \\
\text { Fibrose cística (3) } \\
\text { Desnutrição (1) }\end{array}$ & 4 & 5,2 \\
\hline $\mathrm{K}$ & $\begin{array}{l}\text { Doenças do aparelho digestivo } \\
\text { Apendicite (1) } \\
\text { Doença inflamatória intestinal (1) } \\
\text { Estenose anal pós retocolite (1) } \\
\text { Refluxo gastro-esofágico (1) }\end{array}$ & 4 & 5,2 \\
\hline$P$ & $\begin{array}{l}\text { Algumas afecções originadas no período perinatal } \\
\text { Membrana hialina (1) } \\
\text { Prematuridade (3) }\end{array}$ & 4 & 5,2 \\
\hline $\mathrm{H}$ & $\begin{array}{l}\text { Doenças do olho e anexos } \\
\text { Transtorno dos músculos oculares, movimento binocular } \\
\text { da acomodação e da refração (1) }\end{array}$ & 1 & 1,3 \\
\hline 1 & $\begin{array}{l}\text { Doenças do aparelho circulatório } \\
\text { Miocardiopatia dilatada (1) }\end{array}$ & 1 & 1,3 \\
\hline $\mathrm{N}$ & Doenças do aparelho geniturinário, insuficiência renal crônica & 1 & 1,3 \\
\hline $\mathrm{T}$ & $\begin{array}{l}\text { Sequelas de traumatismos, de intoxicações e de outras } \\
\text { consequências de causas externas } \\
\text { Estenose cáustica do esôfago (1) }\end{array}$ & 1 & 1,3 \\
\hline Z & $\begin{array}{l}\text { Fatores que influenciam o estado de saúde e o contato } \\
\text { com os serviços de saúde } \\
\text { Criança exposta ao vírus da imunodeficiência humana (1) }\end{array}$ & 1 & 1,3 \\
\hline
\end{tabular}

${ }^{a} n=77$. Os valores entre parêntesis representam o número de pacientes com cada uma das condições listadas.

ONGs que assistem crianças em risco social e em tratamento em unidades públicas terciárias de saúde.

Dentre os indicadores sugeridos, aqueles relacionados à internação, (total de internações, percentual de crianças internadas, média de internações por criança, total de dias internados, tempo médio de internação) identificaram mudanças ocorridas após a intervenção. As reduções do total de dias de internação e do número de crianças internadas no período da intervenção contribuíram para a diminuição da média de dias de inter- nação. A diferença absoluta do total de dias de internação entre os dois períodos representou uma medida de eficácia, uma vez que 272 dias de internação foram evitados. Estes resultados sugerem um maior controle das condições clínicas dos pacientes portadores de HIV / AIDS, de doenças do sistema nervoso e malformações congênitas, pois são doenças que tendem a evoluir com um agravamento do quadro clínico. Tal fato reflete em três dimensões: no paciente, na família, na comunidade e nos recursos de saúde. Internações curtas e resolutivas poupam o paciente da privação da convivência familiar, diminuem a ausência no lar do responsável que acompanha o filho na internação e reduzem os riscos inerentes ao ambiente hospitalar $(13,14)$. A comunidade se beneficia com a disponibilidade de serviços para pacientes que não têm acesso a estes serviços em função da baixa rotatividade provocada pelas internações de longa duração e por fim, os recursos economizados podem ser redirecionados a novos programas e tecnologias.

Deve-se considerar que o resultado vai além da soma do efeito de duas intervenções associadas. A parceria entre a instituição pública e a ONG potencializou a efetividade do tratamento provavelmente pela viabilidade de adesão. A melhoria do quadro de saúde das crianças pode trazer oportunidades para superação das condições de vulnerabilidade destas famílias.

Embora toda a população do estudo recebesse assistência médica do IFF e apoio da Refazer, houve diferença entre os pacientes na resposta às intervenções. $\mathrm{Al}$ guns mantiveram a necessidade de internação, mesmo após o apoio da ONG. O estudo buscou identificar quais características dos pacientes poderiam estar contribuindo para a diferença de resultado entre eles. A análise demonstrou que o grupo de pacientes que internou após a entrada na Refazer apresentou maior prevalência de malformações congênitas, doenças neurológicas e HIV / AIDS, sugerindo que a ocorrência de internações pode estar associada a gravidade destas doenças neste grupo. Por outro lado,o grupo de pacientes portadores de doenças pulmonares não internou após a entrada na Refazer, o que pode estar relacionado a uma resposta mais favorável destas patologias ao apoio recebido.

A coleta de informação sobre internações fora do IFF possibilitou evitar um 
TABELA 4. Análise dos indicadores de utilização de serviços hospitalares pelas crianças e adolescentes $^{a}$ em risco social em tratamento no Instituto Fernandes Figueira, Rio de Janeiro, Brasil, e assistidas pela Refazer, 2003-2007

\begin{tabular}{|c|c|c|c|}
\hline Indicador & $\begin{array}{c}\text { Antes } \\
\text { de Refazer }\end{array}$ & $\begin{array}{c}\text { Depois } \\
\text { de Refazer }\end{array}$ & $P$ \\
\hline \multicolumn{4}{|l|}{ Internações } \\
\hline No. total de internações & 50 & 40 & \\
\hline No. de crianças internadas (\%) & $29(39,2)$ & $20(27,0)$ & 0,02 \\
\hline Média de internações por criança (DPb) & $0,7(1,1)$ & $0,5(1,1)$ & 0,24 \\
\hline No. mínimo - No. máximo de internações & $0-5$ & $0-5$ & \\
\hline Total de dias internados & 1010 & 737 & \\
\hline Tempo médio por criança em dias (DP) & $13,6(29,7)$ & $10,0(27,3)$ & 0,02 \\
\hline No. mínimo - No. máximo de dias & $0-147$ & $0-141$ & \\
\hline \multicolumn{4}{|l|}{ Internações na Unidade de Pacientes Graves } \\
\hline No. total de internações & 9 & 6 & \\
\hline No. de crianças internadas (\%) & $8(10,8)$ & $3(4,1)$ & 0,06 \\
\hline Média de internações por criança (DP) & $0,1(0,4)$ & $0,1(0,4)$ & 0,42 \\
\hline No. mínimo - No. máximo de internações & $0-2$ & $0-3$ & \\
\hline Total de dias & 129 & 68 & \\
\hline Média por criança (DP) & $1,7(6,6)$ & $0,9(4,6)$ & 0,16 \\
\hline No. mínimo - No. máximo de dias & $0-39$ & $0-28$ & \\
\hline No. de crianças que usaram tecnologia durante internação (\%) & $21(28,4)$ & $13(17,6)$ & 0,06 \\
\hline \multicolumn{4}{|l|}{ Consultas ambulatoriais } \\
\hline No. total de consultas & 541 & 514 & \\
\hline No. de crianças que realizaram consulta (\%) & $71(92,2)$ & $74(100,0)$ & 0,25 \\
\hline Média por criança (DP) & $7,31(5,1)$ & $6,95(5,0)$ & 0,61 \\
\hline No. mínimo - No. máximo & $0-22$ & $1-22$ & \\
\hline Total de consultas sem sintomas & 225 & 235 & \\
\hline No. de crianças (\%) & $58(78,4)$ & $60(81)$ & 0,81 \\
\hline Média por criança (DP) & $3,0(3,0)$ & $3,2(3,1)$ & 0,77 \\
\hline No. mínimo — No. máximo & $0-13$ & $0-14$ & \\
\hline Total de consultas relacionados à doença de base & 160 & 145 & \\
\hline No. de crianças (\%) & $42(56,8)$ & $40(54)$ & 0,81 \\
\hline Média por criança (DP) & $2,2(2,9)$ & $2,0(3,0)$ & 0,29 \\
\hline No. mínimo - No. máximo & $0-12$ & $0-14$ & \\
\hline Total de consultas com evento sentinela & 19 & 12 & \\
\hline No. de crianças (\%) & $12(16,2)$ & $8(10,8)$ & 0,42 \\
\hline Média por criança (DP) & $0,26(0,7)$ & $0,16(0,5)$ & 0,28 \\
\hline No. mínimo - No. máximo & $0-4$ & $0-3$ & \\
\hline
\end{tabular}

${ }^{a} n=74$.

b DP: desvio padrão.

erro na interpretação dos resultados, no caso da redução das internações na unidade serem compensadas por um acréscimo na procura por outros serviços, apesar de não poder ser descartada a possibilidade de algum sub-registro desta informação. Em ambos os períodos foram poucas as referências às internações fora do IFF, o que poderia ser explicado pelo vínculo que os pacientes crônicos estabelecem com a instituição. $\mathrm{Na}$ vigência da intervenção, ocorreu uma redução dessas internações, o que corrobora com os resultados obtidos para as internações no IFF.

Os indicadores de utilização de serviços ambulatoriais não apresentaram diferenças entre os períodos, mostrando que estes serviços não sofreram reflexos da redução das internações.

Os indicadores de internação identificados neste estudo podem ser úteis para uma avaliação da adequação das ações de ONG que prestam assistência as crianças com doenças crônicas. Segundo Habicht et al. (15) a seleção de indicadores é etapa essencial no processo avaliativo e eles são definidos a partir do modelo lógico-teórico, do fluxo de atividades do programa e dos resultados que se pretende alcançar, sendo os indicadores de resultados aqueles que medem a eficácia e o impacto das ações sobre a população alvo.

Outros estudos que avaliaram ações de apoio ao tratamento de crianças com doenças respiratórias, através de indicadores de utilização de serviços de saúde mostraram resultados semelhantes aos obtidos neste trabalho, isto é, que este tipo de intervenção complementa de forma favorável as ações de saúde, reduzindo o número de internações e o tempo de permanência. Aplicando indicadores como "total de dias de internação" e "procura por atendimento de emergência", Weinstein et al. (16) observaram que crianças portadoras de asma grave, quando incluídas em um programa de apoio ao tratamento, reduziam em $93 \%$ o primeiro indicador e em $81 \%$ o segundo. Mossay et al. (17) observaram um declínio da frequência de internações, do tempo de permanência no hospital e do absenteísmo escolar em crianças portadoras de doença pulmonar crônica inseridas em um projeto multidisciplinar para sua reintegração no âmbito familiar, social e escolar. Bratton et al. (18) avaliaram os resultados de um programa que incorporava a família ao cuidado de crianças portadoras de asma grave, através da comparação com os dados de um estudo realizado dez anos antes da implantação do programa. Os autores detectaram uma redução significativa na duração do tempo de internação das crianças, que diminuiu de uma média de 75 dias para 15,6 dias. Da mesma forma, as crianças portadoras de doenças respiratórias, quando associaram ao tratamento, o apoio da Refazer, não apresentaram internações no período em que receberam o apoio.

Numa revisão da literatura foram identificados apenas quatro publicações que tiveram como foco a análise do resultado de ações desenvolvidas por ONGs na saúde da criança, utilizando como indicadores taxa de mortalidade, taxa de utilização de serviços, cobertura vacinal, taxa de adesão, nível de incorporação de comportamentos e/ou práticas (1). Estes estudos mostraram que a atuação das ONGs teve papel no desempenho de tais indicadores (19-22). Mercer et al. (19) estudaram a eficácia de ações de ONGs em Bangladesh, no período de 1996 a 2002, através da comparação da assistência dada a população geral com a da população de áreas assistidas por ONGs. Os autores encontraram uma maior cobertura de serviços de saúde da criança e um declínio da mortalidade neonatal, pós-natal e infantil nas áreas de atuação das ONGs. Resultado semelhante foi observado por Amin \& Li (21) quando compararam as taxas de mortalidade e de cobertura de imunização de duas populações de Bangladesh. A população habitante de área assistida por ONGs apresentava um desempenho melhor dos indicadores do que a população não assistida. Em um estudo piloto realizado com 22 crianças portadoras de pneumopatia crônica em tratamento no IFF e atendidas pela Refa- 
TABELA 5. Comparação das características dos quatro grupos de pacientes assistidos pela

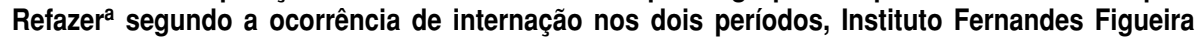
(IFF), Rio de Janeiro, Brasil

\begin{tabular}{|c|c|c|c|c|c|c|c|c|}
\hline \multirow[b]{2}{*}{ Característica } & \multicolumn{2}{|c|}{$\begin{array}{l}\text { Grupo } 1 \\
\text { Não } \\
\text { internou }\end{array}$} & \multicolumn{2}{|c|}{$\begin{array}{l}\text { Grupo } 2 \\
\text { Internou } \\
\text { antes }\end{array}$} & \multicolumn{2}{|c|}{$\begin{array}{c}\text { Grupo } 3 \\
\text { Internou } \\
\text { depois }\end{array}$} & \multicolumn{2}{|c|}{$\begin{array}{c}\text { Grupo } 4 \\
\text { Internou antes } \\
\text { e depois }\end{array}$} \\
\hline & No. & $\%$ & No. & $\%$ & No. & $\%$ & No. & $\%$ \\
\hline Sexo & 43 & 58,1 & 11 & 14,9 & 2 & 2,8 & 18 & 24,2 \\
\hline Feminino & 14 & 32,6 & 5 & 45,5 & 1 & 50,0 & 9 & 50,0 \\
\hline Masculino & 29 & 67,4 & 6 & 54.5 & 1 & 50,0 & 9 & 50,0 \\
\hline Media de idade (DPb) & 7,1 & 4,1 & 7,8 & 4,5 & 5,4 & 5,2 & 5,5 & 4,3 \\
\hline \multicolumn{9}{|l|}{ Diagnóstico } \\
\hline Anomalias congênitas & 12 & 28,0 & 4 & 36,4 & 1 & 50,0 & 7 & 38,9 \\
\hline Doenças do sistema nervoso & 7 & 16,3 & 1 & 9,1 & 1 & 50,0 & 5 & 27,9 \\
\hline HIV & 3 & 7,0 & 1 & 9,1 & & & 2 & 11,1 \\
\hline $\begin{array}{l}\text { Doenças do aparelho } \\
\text { respiratório }\end{array}$ & 11 & 25,5 & 2 & 18,2 & & & & \\
\hline Outros & 10 & 23,2 & 3 & 27,3 & & & 4 & 22,2 \\
\hline \multicolumn{9}{|l|}{ Escolaridade do responsável } \\
\hline$<1^{\circ} \mathrm{grau}$ & 23 & 53,5 & 5 & 45,5 & 1 & 50,0 & 3 & 16,7 \\
\hline $1^{\circ}$ grau completo & 9 & 20,9 & 2 & 18,2 & 11 & 50,0 & 5 & 27,8 \\
\hline$\geq 2^{\circ}$ grau & 6 & 14,0 & 2 & 18,2 & & & 4 & 22,2 \\
\hline Não referido & 5 & 11,6 & 2 & 18,2 & & & 6 & 33,3 \\
\hline $\begin{array}{l}\text { Tempo médio (em dias) de } \\
\text { tratamento no IFF }\end{array}$ & 5,6 & 3,6 & 5,16 & 3,1 & 4,4 & 4,0 & 4,3 & 3,5 \\
\hline
\end{tabular}

zer, Magalhães Costa et al. (23) observaram que $91,7 \%(11 / 12)$ dos pacientes da amostra reduziram o número de internações e o tempo de permanência no período em que passaram a receber o apoio da ONG. Khan (24) comparou os custos da distribuição de vacinas em áreas urbanas de Bangladesh, algumas assistidas por órgãos governamentais e outras por ONGs. Os resultados mostraram que a participação da ONG foi mais eficaz na assistência dada à população alvo como na gestão dos custos do processo de distribuição das vacinas. O baixo peso, o atraso no desenvolvimento e a perda de proteína foram os indicadores utilizados por Senbanjo et al. (22) para avaliar a prevalência de desnutrição em uma área rural da Nigéria. Os autores observaram uma baixa prevalência de desnutrição e atribuíram o fato aos serviços e intervenções providos por ONGs à comunidade. Estes estudos corroboram o resultado encontrado, isto é, que os indicadores de saúde ou de utilização de serviços de saúde são apropriados para avaliar ações de ONG atuantes na área de saúde da criança.

Outra etapa importante no processo de avaliação diz respeito ao grau de inferência - adequação e plausibilidade - que se quer atribuir aos resulta- dos. De acordo com Habicht et al. (15) os estudos de análise de impacto de programas de saúde, abordam três tipos de avaliação: adequação, plausibilidade e probabilidade. A avaliação da adequação informa se os programas estão se desenvolvendo conforme planejado inicialmente, se as mudanças estão ocorrendo na direção e magnitude esperadas, se as metas estão sendo atingidas e se os resultados obtidos estão em consonância com a missão da organização. Se a avaliação não constata mudança nos indicadores, não significa, necessariamente, que o programa não foi eficaz. Deve-se considerar que condições especiais podem interferir na expressão do indicador e na ausência do programa, esses mesmos indicadores poderiam apontar para uma distância ainda maior em relação às metas (25). Esta etapa da avaliação deve preceder as avaliações de plausibilidade.

A avaliação da adequação tem como vantagem a utilização de dados secundários, o que reduz custos do processo de avaliação $(15,25)$. No entanto, existem algumas limitações nos seus resultados, uma vez que não estabelecem uma relação de causa e efeito. Para isto, seria necessário um estudo de plausibilidade no qual é mister a composição de um grupo controle, o que permite uma validação dos resultados. A não realização desta etapa foi uma limitação do presente estudo e deveu-se a baixa comparabilidade das idades e das doenças dos possíveis controles identificados a partir do banco de dados disponível. Outros autores têm relatado dificuldades semelhantes em estudos, cuja intervenção complementa um tratamento de morbidade grave e acabam optando por comparações antes e depois (16-18). Um estudo prospectivo com grupo controle formado por crianças com as mesmas condições daquelas atendidas na ONG, mas que, por alguma restrição, não fossem admitidas nos programas da ONG, comparado ao grupo da intervenção, atenderia ao desenho de um estudo de plausibilidade e permitiria a validação dos resultados. No entanto, para a incorporação de rotinas de avaliação realizadas pela própria equipe da ONG, compor um grupo comparável aleatório é pouco viável, sobretudo pelas questões éticas implicadas num estudo com este desenho.

As características do atendimento da Refazer cumpriram parte importante da metodologia de avaliação, uma vez que não se pode atribuir as mudanças à intervenção sem antes observar a oferta, a utilização e a cobertura do programa. Uma lacuna nesta etapa pode ser considerada como um dos sete pecados capitais nas avaliações de impacto (26). Considerando o atendimento dos itens demandados em relação à população assistida, o apoio da Refazer assemelha-se a parâmetros internacionais de cobertura observados em ONG $(19,21)$.

Estudos de qualidade de vida, custo efetividade, percepção e satisfação do cliente, isto é, dos beneficiados pelas ações, podem complementar a avaliação de impacto e mostrar outras dimensões dos efeitos da intervenção.

A descrição do perfil da população assistida pela Refazer permitiu avaliar que as ações estão sendo dirigidas ao seu público-alvo, um grupo socialmente vulnerável. O baixo nível socioeconômico associado às desigualdades sociais estruturais existentes no país contribuem para manutenção do ciclo vicioso de miséria e doença. O aumento da cobertura das políticas sociais de transferência de renda nos anos mais recentes, pouco atingiu esta população que informou receber somente o BPC. Apesar de este grupo ter acesso gratuito aos serviços de saúde ga- 
rantido pelo SUS, que tem o papel de reduzir a desigualdade social, o apoio à continuidade de onerosos e prolongados tratamentos para graves problemas de saúde, tem sido negligenciado pelas políticas de inclusão social implementadas no país. A mudança de padrão das doenças pediátricas, que adquiriram um perfil de cronicidade, trouxe desafios para as políticas de saúde, pois requer tratamentos complexos e permanentes, o que contribui para a dificuldade desta população em aderir ao tratamento recomendado. É nesta lacuna que atuam as ONGs ao se constituírem numa rede de apoio e ao disponibilizarem medicamentos, equipamentos e recursos para locomoção. Ações que deveriam ser assumidas por políticas públicas de modo a conferir a todos aqueles que necessitam acesso ao tratamento adequado e continuado, estão sendo executadas pela sociedade civil, que tem uma limitação de capacidade de atuação e que não atende a demanda de todos os que estão em condições de vulnerabilidade. Para as famílias pobres, estar inserida em uma rede de apoio pode ser determinante para a adesão das crianças a um tratamento adequado (27).

Pode-se concluir que a metodologia apresentada implicou na descrição das características sociodemográficas e da morbidade da população assistida, descrição das ações desenvolvidas e do grau de atendimento da ONG às demandas dos profissionais de saúde e na análise de adequação através dos resultados dos indicadores antes e depois da intervenção.

A Refazer integra uma rede composta por 23 entidades que realizam atividades similares, voltadas para crianças pacientes de hospitais públicos. A metodologia proposta pode ser uma forma padronizada de avaliações de outras ONGs, cuja população assistida possua características se- melhantes às crianças deste estudo. As organizações poderiam incorporar em seus registros informações relacionadas aos indicadores propostos de modo a acompanhar a evolução dos assistidos, analisar periodicamente os resultados e promover a tomada de decisões e ajustes das ações.

\section{Conclusão}

A análise dos indicadores mostrou que os resultados estão adequados à missão da ONG e a população assistida apresenta um perfil que se beneficia com a inserção na rede de apoio social. Apesar da impossibilidade em se afirmar que os benefícios encontrados são exclusivos às ações da ONG, desenvolveu-se uma avaliação de adequação simples e de fácil aplicação pelas próprias ONGs, o que representa um avanço na direção da institucionalização de avaliações pelas ONGs que atuam na saúde.

\section{REFERÊNCIAS}

1. Magalhães Costa AM, Silveira K, Bonan C. Organizações Não Governamentais na área da Saúde da Criança - Revisão da Literatura. Rev C S Col. 2011;16(7):3181-96.

2. Center for Global Development. When will we ever learn? Closing the Evaluation Gap. Washington (DC). Disponível em www. cgdev.org/section/initiatives/_active/eval gap Acesso em 06 de Junho de 2011.

3. Santos IS, Victora CG. Serviços de Saúde: epidemiologia, pesquisa e avaliação. Cad Saude Publica. 2004;20 Suppl 2:337-41.

4. Rede Saúde Criança Renascer. Disponível em http://www.saudecrianca.org.br/blog/blogda-fundadora/pt-bonecos-da-copa/ Acesso em 06 de junho de 2011.

5. Ministério da Saúde. Norma Operacional da Assistência à Saúde. Sistema Único de Saúde (NOAS/SUS). Portaria ${ }^{\circ} 95$ de 26 de janeiro de 2001. Diário Oficial da União [DOU] 2001 Jan 29; Sec 1, 139 (20E): 23-31.

6. Felisberto E. Monitoramento e avaliação na atenção básica: novos horizontes. Rev Bras Saúde Mater Infant. 2004;4(3):317-21.

7. Ministério da Saúde. Portaria SAS/MS 745, 22 de dezembro de 2005.

8. Instituto Refazer - Grupo de Apoio à Criança e ao Adolescente. Disponível em http:// www.wix.com/refazer/org\#!_base Acesso em 06 de junho de 2011.

9. Soares $\mathrm{S}$. As políticas públicas e a desigualdade racial no Brasil - 120 anos após a abolição. In: Theodoro M, Jaccoud L, Osório RG, Soares S. A demografia da cor: a composição da população brasileira de 1890 a 2007. IPEA: 2008. Pp. 97-117.

10. Ministério do Desenvolvimento Social e Combate a Fome. Disponível em http:// www.mds.gov.br/assistenciasocial/benefi ciosassistenciais/bpc Acesso em 06 de junho de 2011.

11. Centro Colaborador da OMS para a Classificação de Doenças em Português - CBCD. Classificação estatística internacional de doenças e problemas relacionados à saúde. Disponível em http://www.datasus.gov.br/ cid10/v2008/cid10.htm. Acesso em 10 de maio 2010

12. Samico I, Hartz ZM de A, Felisberto E, Frias PG. A sala de situações na Unidade de Saúde da Família: o Sistema de Informação de Atenção Básica (SIAB) como instrumento para o planejamento estratégico local. Saúde Debate. 2002;26(61):236-44.

13. Araújo TBC, Cunha, MAA, Laranjeiras JRF, Gama ACO, Lima DMGF, Lima MCGF. Sistema de internação hospitalar parcial: estudo em 128 crianças com pneumonia. Brasília Med. 2000;37(3/4):76-80.

14. Campos Jr D. Hospitalização parcial de crianças: alternativa para reduzir custos e humanizar o tratamento. Brasília Med. 2000;37 $(3 / 4): 70-1$.

15. Habicht JP, Victora CG, Vaughan JP. Evaluation designs for adequacy, plausibility and probability of public health programme performance and impact. Int J Epidemiol. 1999; 28(1):10-8.

16. Weinstein AG, Faust DS, McKee L, Padman R. Outcome of short-term hospitalization for children with severe asthma. J Allergy Clin Immunol. 1992;90(1):66-75.

17. Mossay C, De Buck C, Filosof D, Parise L. Chronic respiratory pathology in the child: overview of 10 years of multidisciplinary management in a pediatric medical center.
Eur Ann Allergy Clin Immunol. 2003;35(7): 241-6.

18. Bratton DL, Price M, Gavin L, Glenn K, Brenner M, Gelfand EW et al. Impact of a multidisciplinary day program on disease and healthcare costs in children and adolescents with severe asthma: a two-year followup study. Pediatr Pulmonol. 2001;31(3): 177-89.

9. Mercer A, Khan MH, Daulatuzzaman M, Reid J. Effectiveness of an NGO primary health care programme in rural Bangladesh: evidence from the management information system. Health Policy Plan. 2004;19(4):187-98.

20. Chowdhury AM, Karim F, Sarkar SK, Cash RA, Bhuiya A. The status of ORT (oral rehydration therapy) in Bangladesh: how widely is it used? Health Policy Plan. 1997;12(1): 58-66.

21. Amin R, Li Y. NGO-promoted women's credit program, immunization coverage, and child mortality in rural Bangladesh. Women Health. 1997;25(1):71-87.

22. Senbanjo IO, Adeodu OO, Adejuyigbe EA. Low prevalence of malnutrition in a rural Nigerian community. Trop Doct. 2007;37(4): 214-6.

23. Magalhães Costa AM, Silveira K, Dutra MV Reflexo de Ações Sociais Voltadas para a Saúde da Criança sobre Indicadores Hospitalares. In: ABRASCO. Anais VIII Congresso Brasileiro de Saúde Coletiva e XI Congresso Mundial de Saúde Coletiva; 2006 Rio de Janeiro; 2006 CD ROM.

24. Khan MM, Khan SH, Walker D, Fox-Rushby J, Cutts F, Akramuzzaman SM. Cost of delivering child immunization services in urban Bangladesh: a study based on facility-level 
surveys. J Health Popul Nutr. 2004;22(4): 404-12.

25. Santos IS. Avaliação do impacto de programas nutricionais. Rev Nutr. 2009;22(1):141-50.

26. Ministério da Saúde. Avaliação dos programas de saúde é tema de Ciclo de Estudos http://portal.saude.gov.br/portal/saude/ Gestor $/$ visualizar texto.cfm?idtxt $=28553$ Acesso em 06 de Junho de 2011.

27. Bronfman P, Mario N. Multimortalidad y estructura familiar: um estudio cualitativo de las muertes infantiles en las familias [tese]
Rio de Janeiro (RJ): Escola Nacional de Saúde Pública, Fundação Oswaldo Cruz;1993.

Manuscrito recebido em 24 de maio de 2010. Aceito em versão revisada em 26 de fevereiro de 2011

ABSTRACT Objective. Assess the impact of activities by the nongovernmental organization Refazer in support of medical care for children at social risk.

\section{Assessment of the impact of the NGO Refazer on medical care for children at social risk}

Methods. A longitudinal study was conducted of 77 patients from Rio de Janeiro in treatment at the Fernandes Figueira Institute and supported by Refazer. The assessment period was two years, one before and the other during the intervention period while being supported by the NGO. The percentage of hospitalizations, average length of stay, severity of the illness, use of technology, number of outpatient consultations, and clinical conditions involved were compared.

Results. The indicators linked with the hospitalizations revealed changes during the intervention period. There was a reduction in the average time and percentage of hospitalization. The most common pathologies were congenital malformations, chromosomal anomalies, and diseases of the nervous system. These diseases and HIV / AIDS were more prevalent in children that remained hospitalized even after receiving support from the NGO.

Conclusions. Analysis of the indicators showed that the outcomes are adequate to the NGO's mission and that the population helped benefits from participation in the social support network. Although it cannot be stated that the benefits found are due exclusively to the activities of the NGO, a simple and easy-to-use evaluation of adequacy was developed that NGOs themselves can use, representing progress toward the institutional use of evaluations by NGOs working in health.

Key words Non-governmental organizations; program evaluation; child; hospitalization; Brazil. 